\title{
Protocolo largo con análogos de GnRH versus protocolo corto con antagonistas: ¿existen diferencias en cuanto a los resultados de los ciclos de FIV-ICSI?
}

\author{
Laura Blasco G. ${ }^{1}$, Onica Armijo S. ${ }^{1}$, Bárbara Castro M. ${ }^{1}$, Sonia Lobo M. ${ }^{1}$, Carolina \\ González V. ${ }^{1}$, Juan Manuel Montejo G. ${ }^{1}$, M. Carmen Cuadrado M. ${ }^{1}$, Beatriz Fernán- \\ dez M. ${ }^{2}$.
}

${ }^{1}$ Servicio de Reproducción, ${ }^{2}$ Servicio de Bioestadística. Hospital Universitario La Paz. Madrid. España.

\section{RESUMEN}

Objetivo: Valorar si existen diferencias en los resultados de los ciclos de FIV-ICSI en función del protocolo de estimulación empleado. Método: Estudio retrospectivo descriptivo de pacientes infértiles que fueron sometidas a ciclos de FIV-ICSI en el Hospital Universitario La Paz, entre los meses de enero y septiembre de 2010, comparando un protocolo largo de estimulación con análogos de GnRH vs un protocolo corto con antagonistas de $\mathrm{GnRH}$. Las variables analizadas fueron: tasa de gestación, necesidad de cancelación del ciclo, dosis total de gonadotropinas requerida durante la estimulación, niveles de estradiol sérico el día de la administración de la hCG, número de folículos puncionados, complejos obtenidos, número de ovocitos maduros y de embriones conseguidos. Resultados: No hubo diferencias estadísticamente significativas en los resultados de los ciclos en función del protocolo de estimulación empleado, en ninguna de las variables analizadas. Conclusiones: Este estudio no encontró diferencias en los resultados de los ciclos de FIVICSI con relación al uso de análogos o antagonistas de GnRH. Es necesarios más estudios con mayores tamaños muestrales para definir qué tipo de pacientes serían subsidiarias de recibir cada tratamiento para conseguir resultados óptimos.

\section{PALABRAS CLAVE: Análogos o agonistas de GnRH, antagonistas de GnRH, tasa de gestación}

\section{SUMMARY}

Aims: To assess if there exist any differences in the results of the IVF-ICSI cycles depending on the stimulation protocol employed. Methods: Retrospective descriptive study of infertile patients who underwent IVF-ICSI cycles at La Paz University Hospital, between January and September 2010, comparing sitmulation protocol with $\mathrm{GnRH}$ agonists vs antagonists of $\mathrm{GnRH}$. The variables analyzed were pregnancy rate, cancellation rate, total dose of gonadotropin required for stimulation, serum estradiol levels on the day of hCG administration, number of follicles punctured, complexes obtained, number of mature oocytes and of embryos obtained. Results: No statistically significant differences where found in the results of cycles depending on the protocol of stimulation used in any of the variables analyzed. Conclusions: This study didn't find any difference in the outcome of IVF-ICSI cycles in relation to the use of GnRH agonists or antagonists. We need more studies with larger sample sizes to determine which is the best treatment to each patient in order to achieve optimal results.

KEY WORDS: GnRH analogues or agonists, GnRH antagonists, pregnancy rate 


\section{INTRODUCCIÓN}

Los análogos de la hormona liberadora de gonadotropinas $(\mathrm{GnRH})$ tienen capacidad de unirse con mayor afinidad al receptor de $\mathrm{GnRH}$ de forma que producen una supresión hipofisaria tras un aumento inicial en la liberación de gonadotropinas (flare up). Las ventajas que presentan este tipo de fármacos es que minimizan los picos prematuros de $\mathrm{LH}$, disminuyen el número de ciclos cancelados por ovulaciones endógenas, permiten programar la fecha de la punción folicular y consiguen un desarrollo folicular sincrónico. Sin embargo, su uso provoca insuficiencia del cuerpo lúteo, presentan mayores tasas de cuadros de hiperestimulación ovárica y hacen necesario el uso de mayores dosis de gonadotropinas (1).

Los antagonistas de la GnRH actúan bloqueando el receptor de $\mathrm{GnRH}$, produciendo una supresión rápida y profunda y evitando el efecto flare up y permitiendo, de esta forma, comenzar la estimulación ovárica coincidiendo con el reclutamiento folicular.

Actualmente existe controversia en cuanto a si los resultados de los ciclos de fecundación in vitro (FIV) son diferentes en función del protocolo de estimulación empleado (2). El objetivo de este estudio es valorar la existencia de estas diferencias.

\section{MATERIAL Y MÉTODOS}

Pacientes. Se analizan un total de 264 ciclos de FIV-ICSI realizados en el Servicio de Reproducción del Hospital Universitario La Paz, entre los meses de enero y septiembre de 2010. Los criterios de inclusión en cuanto a edad fueron pacientes menores de 40 años; IMC entre 18 y $35 \mathrm{Kg} / \mathrm{m} 2$; número de ciclos previos de FIV-ICSI menor de 2.

Protocolo de estimulación. Se dividió a las pacientes en dos grupos según el protocolo de estimulación que habían recibido. En el primer grupo se incluyó a las pacientes que habían seguido un protocolo largo con análogos de GnRH (desensibilización hipofisaria con Triptorelina $0,1 \mathrm{mg} / \mathrm{día}$ desde el día 22 del ciclo previo y estimulación con FSH desde el $3^{\circ}$ día del ciclo). En el segundo grupo se incluyó a las pacientes que habían seguido un protocolo corto con antagonistas de GnRH (estimulación con FSH desde el $3^{\circ}$ día de ciclo añadiendo Cetrorelix o Ganirelix $0,25 \mathrm{mg}$ a partir del 6음 o cuando se comprobaba por ecografía la presencia de al menos 1 folículo dominante mayor de $14 \mathrm{~mm}$ ).

Determinaciones hormonales. Se analizaron los valores de $\mathrm{FSH}$ y hormona antimülleriana $(\mathrm{AMH})$ previos a iniciar el ciclo de estimulación y el estradiol sérico el día de la punción.
Análisis estadístico. El análisis estadístico se realizó utilizando el programa SPSS. Los datos se expresaron como media \pm desviación estándar $( \pm$ $D E$ ). Para detectar diferencias estadísticamente significativas se aplicaron los test de Chi-cuadrado, $\mathrm{U}$ de Mann-Whitney y $\mathrm{W}$ de Wilcoxon. Consideramos significación estadística un valor $p<0,05$.

\section{RESULTADOS}

En el Servicio de Reproducción del Hospital Universitario La Paz, entre los meses de enero y septiembre de 2010 se realizaron 264 ciclos de FIV-ICSI. La mediana de edad de las pacientes fue de 35,42 años (rango: 23 a 40 años). Del total de ciclos de estimulación realizados, en 116 se utilizó un protocolo de estimulación largo con agonistas de $\mathrm{GnRH}$ y en los 148 restantes un protocolo corto con antagonistas de $\mathrm{GnRH}$. Aunque se objetivó una diferencia (estadísticamente significativa) de 1 año en la media de edad de las pacientes en ambos grupos $(34,84$ años en el grupo que utilizaron agonistas, frente a 35,86 años en el grupo que utilizaron antagonistas) no se observaron diferencias en lo referente a la reserva ovárica documentada como número de folículos antrales, FSH basal y $\mathrm{AMH}$. Este hecho apoya la homogeneidad de los grupos y da consistencia a los resultados. Se consiguieron un total de 80 embarazos, lo que supone una tasa de gestación del $30,3 \%$.

Tasa de gestación según protocolo de estimulación: No se encontraron diferencias estadísticamente significativas en las tasas de gestación en función del protocolo de estimulación utilizado $(p=0,892)$. La tasa de gestación en el grupo en el que se realizó el protocolo largo con agonista fue de $32,7 \%$ y de $31,9 \%$ en el grupo de protocolo corto con antagonistas.

Tasa de cancelación de ciclo según protocolo de estimulación. No se encontraron diferencias estadísticamente significativas $(p=0,437)$ en el número de cancelaciones ajustadas por protocolo de estimulación. En el grupo del protocolo largo con agonistas se cancelaron un $4,3 \%$ de los ciclos mientras que en el grupo de protocolo corto con antagonistas se cancelaron un $7,4 \%$ de los ciclos. Se observa un discreto incremento en el número de cancelaciones en el caso de aplicación de un protocolo corto con antagonistas, pero debido a que el número total de cancelaciones fue muy bajo (16 cancelaciones), estas diferencias no alcanzan significación estadística.

Otros parámetros: En cuanto a la dosis total de FSH requerida para conseguir la estimulación, ésta fue mayor en el grupo en el que se utilizaron análo- 
gos de $\mathrm{GnRH}$, aunque estas diferencias no fueron estadísticamente significativas $(p=0,663)$. Tampoco se encontraron diferencias en los niveles de estradiol sérico el día de la punción $(p=0,249)$, aunque estas fueron ligeramente superiores en los casos en los que se había realizado un protocolo largo. No se encontraron diferencias en cuanto al número de folículos puncionados $(p=0,204)$, número de complejos obtenidos $(p=0,907)$, número de ovocitos maduros $(p=0,615)$ y número de embriones conseguidos $(p=0,787)$ (Tabla I).

Reserva ovárica. Se observó una mayor tasa de gestación en función de la reserva ovárica (edad, FSH basal, AMH y número de folículos antrales) independientemente del protocolo de estimulación utilizado.

\section{DISCUSIÓN}

Existen tres tipos de protocolos en los que se usan agonistas de $\mathrm{GnRH}$. El protocolo largo (el agonista se administra desde la fase lútea del ciclo previo), el protocolo corto (administrando el agonista entre el primer y el tercer día de ciclo, aprovechando el efecto flare up) y el ultracorto (sólo se administra el agonista durante los tres primeros días del ciclo).

También existen dos protocolos diferentes en los que se utilizan antagonistas de $\mathrm{GnRH}$. El protocolo de dosis múltiples, en el que la administración del antagonista comienza en el sexto día de estimulación ovárica o cuando existe un folículo de al menos $14 \mathrm{~mm}$ de diámetro y se continúa hasta el día de la administración de la hCG. La dosis utilizada es de $0,25 \mathrm{mg} / \mathrm{día}$. En el protocolo de dosis única sólo se administra una dosis de GnRH cuando el folículo mayor tiene $14 \mathrm{~mm}$ de diámetro, con una dosis mínima de $3 \mathrm{mg}$.

En éste estudio únicamente hemos comparado los resultados de los ciclos de FIV-ICSI en los casos que se utilizaron los protocolos largos con análogos de GnRH y corto de dosis múltiple con antagonistas.

No existe consenso en lo publicado en cuanto a las tasas de gestación en función del protocolo de estimulación utilizado. En este estudio no hemos observado diferencias estadísticamente significativas. Este resultado concuerda con los obtenidos en la mayoría de las publicaciones (3-7). Sin embargo, existen otros estudios en los que se han observado menores tasas de gestación, en los casos en los que se había utilizado un protocolo de estimulación con antagonistas de GnRH (8).

En gran parte de las publicaciones se documentan diferencias en cuanto a las características del ciclo: mayor duración del tratamiento, mayor dosis de gonadotropinas, y niveles de estradiol más altos el día de la administración de la hCG en los grupos en los que se utilizó un protocolo con agonistas de $\mathrm{GnRH}(6,8,9)$. En nuestro estudio, aunque las diferencias en estos aspectos no fueron estadísticamente significativas, sí se observó una tendencia a requerir mayores dosis de gonadotropinas y valores de estradiol más altos el día de la administración de la hCG, en el grupo en el que se utilizaron agonistas de $\mathrm{GnRH}$. Probablemente, si se aumentara el tamaño muestral, estas diferencias podrían llegar a la significación estadística.

En el estudio de Depalo y cols, publicado en 2009 (5), se documentó un mejor crecimiento folicular y maduración ovocitaria en los casos en los que se había utilizado un protocolo con agonistas de $\mathrm{GnRH}$. Nosotros no hemos observado diferencias estadísticamente significativas a este respecto. Sin embargo, aunque sin alcanzar la significación estadística, el número de ovocitos maduros fue mayor en el grupo en el que se utilizaron antagonistas de GnRH pero con un número menor de embriones.

La mayoría de los estudios están de acuerdo en que la incidencia de síndrome de hiperestimulación ovárica moderado-severo es menor en las pacientes en las que se utilizan antagonistas de $\mathrm{GnRH}$

Tabla I

OTROS PARÁMETROS EN EVALUACIÓN SEGÚN PROTOCOLO DE ESTIMULACIÓN

\begin{tabular}{lccr}
\hline & Agonista largo & Antagonista corto & Valor p \\
\hline Dosis total FSH $(\mathrm{UI})$ & $2198,29 \pm 868,38$ & $2117,52 \pm 798,52$ & 0,663 \\
Estradiol-punción $(\mathrm{pg} / \mathrm{ml})$ & $1839,47 \pm 1439,98$ & $1585,52 \pm 1183,79$ & 0,249 \\
No folículos puncionados & $12,43 \pm 6,79$ & $11,44 \pm 7,17$ & 0,204 \\
No complejos obtenidos & $9,17 \pm 4,91$ & $9,6 \pm 5,76$ & 0,907 \\
N $^{\circ}$ ovocitos maduros & $7,63 \pm 4,5$ & $8,04 \pm 4,99$ & 0,615 \\
No embriones $^{0}$ & $4,96 \pm 3,74$ & $4,93 \pm 3,5$ & 0,787 \\
\hline
\end{tabular}


$(6,8,10)$. Esto puede ser debido a la opción de desencadenar la ovulación con un agonista de GnRH en los casos en los que se sospeche un riesgo elevado de que se produzca un síndrome de hiperestimulación ovárica. En este estudio no se observaron diferencias en lo que se refiere a cancelaciones de ciclo por riesgo de hiperestimulación ovárica en función del tipo de protocolo utilizado.

\section{CONCLUSIÓN}

En este estudio no se encontró diferencias en cuanto a los resultados de los ciclos de FIV-ICSI con relación al uso de análogos o antagonistas de $\mathrm{GnRH}$. Los resultados publicados en la literatura son contradictorios en lo que se refiere a las tasas de gestación, aunque existen más publicaciones que argumentan que no existen diferencias. Probablemente sean necesarios más estudios y con mayores tamaños muestrales para definir qué tipo de pacientes serían subsidiarias de recibir cada tratamiento para conseguir resultados óptimos.

\section{BIBLIOGRAFÍA}

1. Remohí J, Bellver J, Domingo J, Bosch E, Pellicer A. Manual práctico de esterilidad y reproducción humana. Mc Graw Hill. 2008.

2. Huirne JA, Homburg R, Lambalk CB. Are GnRH antagonists comparable to agonists for use in IVF? Hum Reprod 2007;22:2805-13.

3. Bodri D, Sunkara SK, Coomarasamy A. Gonadotropin-releasing hormone agonists versus antagonists for controlled ovarian hyperestimulation in oocyte donors: a systematic review and meta-analisys. Fertil Steril 2011;95:164-9.

4. Devesa M, Martínez F, Coroleu B, Tur R, González C, Rodríguez I, Parri PN. Poor prognosis for ovarian response to stimulation: results of a randomised trial comparing de flare-up $\mathrm{GnRH}$ agonst protocol vs. the antagonist protocol. Gynecol Endocrinol 2010;26:509-15.

5. Depalo R, Lorusso F, Palmisano M, Bassi E, Totaro I, Vacca M, Trerotoli P, Masciandaro P, Selvaggi L. Follicular growth an oocyte maturation in $\mathrm{GnRH}$ agonist and antagonist protocols for in vitro fertilization and embryo transfer. Gynecol Endocrinol 2009;25:328-34.

6. Lainas TG, Sfontouris IA, Zorzovilis IZ, Petsas GK, Lainas GT, Alexopoulou E, Kolibianakis EM. Flexible $\mathrm{GnRH}$ antagonist protocol vs $\mathrm{GnRH}$ agonist long protocol in patients with polycystic ovary syndrome treated for IVF: a prospective randomized controlled trial (RTC). Hum Reprod 2010;25:683-9.

7. Engel JB, Griesinger G, Schultze-Mosgau A, Felberbaum R, Diedrich K. GnRH agonists and antagonists in assisted reproduction: pregnancy rate. Reprod Biomed Online 2006;13:84-7.

8. Al-Inany HG, Abou-Setta AM, Aboulghar M. Gonadotropin-releasing hormone antagonists for assisted conception. Cochrane Database Syst Rev. 2006 Jul 19;3:CD001750.

9. Hosseini MA, Aleyasin A, Saeedi H, Mahdavi A. Comparison of gonadotropin-releasing hormone agonists and antagonists in assisted reproduction cycles of polycystic ovarian syndrome patients. J Obstet Gynaecol Res 2010;36:605-10.

10. Streda R, Mardesic T, Sobotka V, Tosner J. Long $\mathrm{GnRH}$ agonist vs. GnRH antagonist protocol in randomized controlled trial in unselected patients-hormonal and cycle characteristics-pilot study. Ceska Gynekol 2009;74:75-80. 